\title{
Iterative Approximation for a Boundary Value Problem Arising for the Electrical Potential on a Cylindrical Double Layer
}

\author{
Jürgen Appell, Chur-jen Chen, Shiojenn Tseng, \\ and Martin Väth
}

\begin{abstract}
Building on Banach's contraction mapping principle, we give existence, uniqueness, and approximation theorems for a boundary value problem on an unbounded domain arising in the mathematical modelling of the electrical potential in a cylindrical double layer.
\end{abstract}

Keywords. Poisson-Boltzmann equation, electric potential distribution, modified Bessel functions, weighted function spaces, contraction mapping principle

Mathematics Subject Classification (2000). 35J65, 35Q60, 33C10, 47H10

\section{Introduction and statement of the problem}

Schauder's and Banach's fixed point theorems belong to the most useful tools for proving existence and uniqueness results for various problems arising in mechanics, physics, engineering, chemistry, and biology. To cite a recent sample result, in [5] it was shown by the authors that both fixed point principles may be used successfully to study the mathematical model for an adiabatic tubular chemical reactor which processes an irreversible exothermic reaction. One should remark, however, that Banach's fixed point principle is more popular among application-oriented scientists, because it is, in contrast to Schauder's

Jürgen Appell: University of Würzburg, Department of Mathematics, Am Hubland 9, D-97074 Würzburg, Germany; jurgen@dmuw.de

Chur-Jen Chen: Tunghai University, Department of Mathematics, Taichung 407, Taiwan, RP China; ccjj@math.thu.edu.tw

Shiojenn Tseng: Tamkang University, Department of Mathematics, Tamsui 251, Taipei, Taiwan, RP China; tseng@math.tku.edu.tw

Martin Väth: Free University of Berlin, Department of Mathematics (WE1), Arnimallee 2-6, D-14195 Berlin, Germany; vaeth@mathematik.uni-wuerzburg.de 
theorem, constructive and hence makes it possible to obtain iterative approximations which are arbitrarily close to the exact (and often unknown) solution.

Another field where this may be successfully applied is chemical engineering, see e.g. [1, 2, 8]. For instance, in [8] the authors study the electric potential distribution for the case of an infinitely long cylindrical surface which is basically determined by the concentrations and the valences of the ionic species in the liquid phase, the radius of the surface, the length of particles, the relative permittivity of the liquid phase, the temperature, and the potential on the given surface. Since this problem has been our main motivation for this paper, we would like to describe it at some length in what follows.

The mathematical modelling of this problem leads to the classical PoissonBoltzmann equation [2]

$$
\Delta_{r} \psi(r)=-\frac{\rho(r)}{\varepsilon}
$$

for the unknown electric potential $\psi=\psi(r)$, where $\rho=\rho(r)$ denotes the space charge density in the diffuse double layer, and $\varepsilon$ is the relative permittivity of the medium. If the potential $\psi$ is considered outside a nonconductive infinitely long cylinder of radius $R>0$ immersed in an electrolyte solution, evaluating the radial Laplace operator $\Delta_{r}$ in (1.1) in polar coordinates yields

$$
\frac{d}{d r}\left(r \frac{d \psi(r)}{d r}\right)=-\frac{r \rho(r)}{\varepsilon} \quad(r>R)
$$

The natural boundary conditions associated with this equation are

$$
\psi(R)=\psi_{0}, \quad \lim _{r \rightarrow \infty} \psi(r)=0,
$$

where $\psi_{0}$ is the given potential on the cylindrical surface. For the case of a $z: z$ electrolyte with a number concentration $n_{0}$, equation (1.1) becomes

$$
\Delta_{r} \psi(r)=\frac{2 z e n_{0}}{\varepsilon} \sinh \left(\frac{z e \psi(r)}{k_{B} T}\right) \quad(r>R),
$$

where $e$ is the elementary charge, $z$ is the valence of bulk ions, $k_{B}$ is the Boltzmann constant, and $T$ is the temperature. Note that (1.3) is a nonlinear second order elliptic equation with a continuous odd nonlinearity on the right hand side; this fact will be important for our mathematical analysis in the sequel. Under the Debye-Hückel condition [2], i.e., assuming $\psi(r)$ to be low, the solution to equation (1.3), subject to the initial condition $\psi(R)=\psi_{0}$ from (1.2), may be written in the form

$$
\psi(r)=A K_{0}(\kappa r) \quad(r>R),
$$

where $A$ is some constant, $\kappa$ is the reciprocal Debye length, and $K_{0}$ denotes the modified zeroth order Bessel function of second kind (see below). 
If we denote by $F$ the nonlinear Nemytskij operator generated by the hyperbolic sine function in the right hand side of (1.3), i.e., $F(\psi)=f \circ \psi$ with $f(u)=\alpha \sinh (a u)$, where $\alpha:=\frac{1}{\varepsilon} 2 z e n_{0}$ and $a:=\frac{z e}{k_{B} T}$, we may rewrite (1.3) equivalently as operator equation

$$
\Delta_{r} \psi=F(\psi) .
$$

Since the hyperbolic sine function is a homeomorphism of the real line, it is a tempting idea to invert the operator $F$ in a suitable function space und to rewrite (1.5) as fixed point equation

$$
\psi=F^{-1}\left(\Delta_{r} \psi\right)
$$

as was done in the recent paper [8]. However, in this connection one encounters serious technical problems. First, in order to apply the Banach contraction mapping principle one has to find a Banach space such that the operator on the right hand side of (1.6) satisfies a Lipschitz condition

$$
\left\|F^{-1}\left(\Delta_{r} \phi\right)-F^{-1}\left(\Delta_{r} \psi\right)\right\| \leq q\|\phi-\psi\|
$$

in the norm of this Banach space. Second, one has to verify that the Lipschitz constant $q$ in (1.7) may be chosen strictly less than 1. Third, for constructing approximate solutions one has to iterate the operator in (1.6), starting from a carefully chosen initial guess, and to calculate the distance between subsequent iterates. For the fixed point problem (1.6) this is extremely difficult, because the operator on the right hand side does not map any of the "usual" Banach spaces (like spaces of continuous or differentiable functions) into itself. (In the paper [8] the authors use the maximum norm in (1.7) and give only a lower estimate for the constant $q$ in (1.7) by comparing the first two iterates.)

Therefore it seems a better device to consider the inverse of the differential operator $\Delta_{r}$ in (1.5), rather than of the Nemytskij operator $F$. Basically, this amounts to finding a suitable Green's function for the operator $\Delta_{r}$, and to looking for a Banach space in which the operator generated by this Green's function is "well behaved". This is the purpose of the present paper which is organized as follows. In the following section, we recall what is known about existence and uniqueness of solutions of linear boundary value problems for the Laplace operator; this amounts to determining the Green's function of $\Delta_{r}$ (or rather $\Delta_{r}-c^{2}$ id for some constant $c$ ) involving modified Bessel functions of the first and second kind with "suitable" asymptotic behaviour. Once we have determined, at least in principle, the right inverse of the Laplace operator, subject to the boundary conditions (1.2), we may study the nonlinear problem (1.5) by means of Banach's contraction mapping principle. In the last section we return to the sinh-type nonlinearity (1.3) to apply our abstract results to the specific problem given in the title. 


\section{The linear boundary value problem}

Motivated by the problem considered in the introduction, we are interested in the existence, uniqueness, and approximation of the solution of the boundary value problem

$$
\Delta_{r} u(r)=f(u(r))
$$

on an unbounded domain $[R, \infty)$ with boundary values

$$
u(R)=u_{0}, \quad \lim _{r \rightarrow \infty} u(r)=0 .
$$

As before, $\Delta_{r}$ denotes the radial Laplace operator

$$
\Delta_{r} u(r)=\frac{1}{r} \frac{d}{d r}\left(r \frac{d u(r)}{d r}\right)
$$

and $f: \mathbb{R} \rightarrow \mathbb{R}$ is a given $C^{1}$ function such that $f(0)=0$ and $f^{\prime}(0)>0$. As mentioned before, we are mainly interested in the case

$$
f(u)=\alpha \sinh (a u) \quad(\alpha, a>0)
$$

which is motivated by (1.3), or in the more general nonsymmetric case

$$
f(u)=\alpha\left(e^{a u}-e^{-b u}\right) \quad(\alpha, a, b>0) .
$$

However, our theory applies to any other $C^{1}$ function $f$ (with $f(0)=0$ and $f^{\prime}(0)>0$ ) as well. Actually, our approach can also be used if $-f^{\prime}(0) \geq 0$ is small enough, but the results are in such a case typically only useful if $-f^{\prime}$ remains small in a large neighborhood of 0 . We make some remarks on this later.

The main idea to solve problem $(2.1) /(2.2)$ is to rewrite it as a fixed point equation. This can be done by inverting the nonlinear $f$ as was proposed in [8], but it seems that a better approach is to invert $\Delta_{r}$ instead, taking the boundary condition (2.2) into account. We will even invert $\Delta_{r}-c^{2}$ id where $c^{2}$ id approximates in a sense the linearization of $f$ at 0 , i.e., $c \approx \sqrt{f^{\prime}(0)}$. This means that the successive iterations for the corresponding fixed point problem can be considered as approximations in a simplified Newton method, and so the convergence is faster the closer the approximations come to 0 . Roughly speaking, for a small boundary value $u_{0}$ in (2.2) the convergence will usually be much faster than our theoretical bound below; we will see the reason for this later. We point out that one might also consider our approach as a variant of Schauder's linearization trick.

For the case that $f^{\prime}(0)=0$ or $f^{\prime}(0)<0$, one is usually better advised to invert $\Delta_{r}$ or $\Delta_{r}+c^{2}$ id with $c \approx \sqrt{-f^{\prime}(0)}$; however, the latter would mean in our calculations below that we would have to consider classical Bessel functions instead of modified Bessel functions. Since these functions have a rather different asymptotic behaviour, the computations (and results) would also be 
rather different. Therefore, we restrict ourselves in this paper to the inversion of $\Delta_{r}-c^{2}$ id with $c>0$ which, roughly speaking, corresponds to the case $f^{\prime}(0)>0$ and thus is relevant for the physical problem mentioned in the introduction.

The hardest problem in the above approach is to find a suitable function space to invert the operator $\Delta_{r}-c^{2}$ id. It is natural to consider a continuous weight function $w:[R, \infty) \rightarrow(0, \infty)$ satisfying

$$
\lim _{r \rightarrow \infty} w(r)=\infty
$$

and to work in the function space $C_{w}([R, \infty))$ which consists, by definition, of all continuous functions $u:[R, \infty) \rightarrow \mathbb{R}$ for which the weighted norm

$$
\|u\|_{w}:=\sup _{r \geq R}|u(r) w(r)|
$$

is finite. Surprisingly, we will see soon that the only reasonable weight function turns out to be $w=\mathrm{id}$; in this case, we simply write $\|u\|$ instead of $\|u\|_{\text {id }}$ and $C([R, \infty))$ instead of $C_{\text {id }}([R, \infty))$.

To understand this, let us first calculate the right inverse of the operator $\Delta_{r}-c^{2}$ id $(c>0)$ under the boundary assumptions (2.2), i.e., we want to associate to each right hand side $y \in C_{w}([R, \infty))$ a solution $u$ of the problem

$$
\Delta_{r} u-c^{2} u=y
$$

satisfying the additional conditions (2.2). As is well known, a fundamental system of the homogeneous problem corresponding to (2.7) consists of the functions $r \mapsto I_{0}(c r)$ and $r \mapsto K_{0}(c r)$, where

$$
I_{\alpha}(x):=\sum_{k=0}^{\infty} \frac{1}{k ! \Gamma(\alpha+k+1)}\left(\frac{x}{2}\right)^{\alpha+2 k}
$$

and

$$
K_{\alpha}(x):=\frac{\pi}{2} \lim _{\beta \rightarrow \alpha} \frac{I_{-\beta}(x)-I_{\beta}(x)}{\sin \beta \pi}
$$

denote the modified Bessel functions of the first and second kind, respectively, of order $\alpha[3,7,9]$. The function (2.9) is also called Macdonald or Basset function in the literature.

The classical variation-of-constants formula thus shows that the general solution of (2.7) has the form

$$
u(r)=\left(\alpha+\int_{R}^{r} K_{0}(c t) y(t) t d t\right) I_{0}(c r)+\left(\beta-\int_{R}^{r} I_{0}(c t) y(t) t d t\right) K_{0}(c r),
$$

where $\alpha$ and $\beta$ are arbitary constants. 
Recall that the modified Bessel functions (2.8) and (2.9) are nonnegative and have for large arguments the asymptotic behaviour

$$
I_{\alpha}(r)=\frac{\exp r}{\sqrt{2 \pi r}}\left(1+O\left(r^{-1}\right)\right) \quad(r \rightarrow \infty)
$$

and

$$
K_{\alpha}(r)=\frac{\pi \exp (-r)}{\sqrt{2 \pi r}}\left(1+O\left(r^{-1}\right)\right) \quad(r \rightarrow \infty)
$$

respectively $[4,6,9]$. If the weight function $w$ is increasing sufficiently fast, then $y \in C_{w}([R, \infty))$ is decreasing sufficiently fast, and so one should expect that

$$
\int_{R}^{\infty} K_{0}(c t)|y(t)| t d t<\infty
$$

and

$$
\lim _{r \rightarrow \infty} K_{0}(c r) \int_{R}^{r} I_{0}(c t) y(t) t d t=0 .
$$

Under this hypothesis, since $I_{0}(c r) \rightarrow \infty$ and $K_{0}(c r) \rightarrow 0$ as $r \rightarrow \infty$, we can only have $u(r) \rightarrow 0$ as $r \rightarrow \infty$ if

$$
\alpha=-\int_{R}^{\infty} K_{0}(c t) y(t) t d t
$$

Inserting the boundary value $u(R)=u_{0}$, we conclude that $u=A y$, where the operator $A$ is defined by

$$
\begin{aligned}
A y(r):= & -I_{0}(c r) \int_{r}^{\infty} K_{0}(c t) y(t) t d t-K_{0}(c r) \int_{R}^{r} I_{0}(c t) y(t) t d t \\
& +\frac{K_{0}(c r)}{K_{0}(c R)}\left(u_{0}+I_{0}(c R) \int_{R}^{\infty} K_{0}(c t) y(t) t d t\right) .
\end{aligned}
$$

For further reference we summarize with the following

Proposition 2.1. Let $w$ be such that each $y \in C_{w}([R, \infty))$ satisfies (2.12) and (2.13). Then, for each $y \in C_{w}([R, \infty))$, the function $u=A y$ in (2.14) is the only solution of problem (2.7) with boundary values (2.2).

Proposition 2.1 shows that the operator $A$ defined by (2.14) is right-inverse to the operator $\Delta_{r}-c^{2}$ id. It is not too surprising that $A$ is not linear but affine, because we impose nonhomogeneous boundary conditions. To analyze the properties of $A$, we consider the quantity

$$
L_{w}(c, R):=\sup _{r \geq R}\left[M_{w}(c, r)+N_{w}(c, r, R)+\frac{I_{0}(c R)}{K_{0}(c R)} P_{w}(c, r, R)\right],
$$


where we use the shortcuts

$$
\begin{aligned}
M_{w}(c, r) & :=I_{0}(c r) w(r) \int_{r}^{\infty} K_{0}(c t) \frac{t}{w(t)} d t \\
N_{w}(c, r, R) & :=K_{0}(c r) w(r) \int_{R}^{r} I_{0}(c t) \frac{t}{w(t)} d t,
\end{aligned}
$$

and

$$
P_{w}(c, r, R):=K_{0}(c r) w(r) \int_{R}^{\infty} K_{0}(c t) \frac{t}{w(t)} d t
$$

In order to verify that (2.12) and (2.13) hold, and that the operator $A$ maps the space $C_{w}([R, \infty))$ into itself, with good estimates for $\|A x-A y\|$, it will be crucial that the quantity $(2.15)$ be finite. Moreover, the estimates are better the smaller (2.15) is. Hence, for our later considerations, one should choose $w$ in such a way that all terms (2.16), (2.17) and (2.18) are small. The following lemma shows that the weight function $w(r):=r$ is good in this sense.

Lemma 2.2. For $w=\mathrm{id}$, the equality

$$
L_{\mathrm{id}}(c, R)=\frac{1}{c^{2}} L_{\mathrm{id}}(1, c R) \quad(c, R>0)
$$

is true. Morover, there is a universal finite smallest constant $L>1$ such that, for all $c, R>0$, one has

$$
L_{\mathrm{id}}(c, R) \leq \frac{L}{c^{2}}
$$

Finally,

$$
\begin{gathered}
\lim _{r \rightarrow \infty}\left[M_{\mathrm{id}}(c, r)+N_{\mathrm{id}}(c, r, R)+\frac{I_{0}(c R)}{K_{0}(c R)} P_{\mathrm{id}}(c, r, R)\right]=\frac{1}{c^{2}}, \\
\frac{1}{c^{2}} \leq \liminf _{R \rightarrow \infty} L_{\mathrm{id}}(c, R) \leq \limsup _{R \rightarrow \infty} L_{\mathrm{id}}(c, R) \leq \frac{3}{2 c^{2}},
\end{gathered}
$$

and

$$
\limsup _{r \rightarrow \infty} K_{0}(c r) w(r)<\infty .
$$

Proof. The substitution rule for integrals implies easily that

$$
M_{\text {id }}(c, r)=\frac{M_{\text {id }}(1, c r)}{c^{2}}, N_{\text {id }}(c, r, R)=\frac{N_{\text {id }}(1, c r, c R)}{c^{2}}, P_{\text {id }}(c, r, R)=\frac{P_{\text {id }}(1, c r, c R)}{c^{2}},
$$

and thus formula (2.19) follows. For the other claims, it is hence no loss of generality (using again the substitution rule) to assume $c=1$. 
Since (2.11) implies that $r K_{0}(r) \rightarrow 0$, as $r \rightarrow \infty$, the limit relation (2.21) follows if we can show that

$$
\lim _{r \rightarrow \infty} M_{\mathrm{id}}(1, r)=\frac{1}{2}=\lim _{r \rightarrow \infty} N_{\mathrm{id}}(1, r, R) .
$$

To see this, we note first that the asymptotic formulas (2.10) and (2.11) imply that, for $r \rightarrow \infty$,

$$
\begin{gathered}
\frac{K_{\alpha}(r)}{K_{\beta}(r)}=\frac{\frac{\pi \exp (-r)}{\sqrt{2 \pi r}}\left(1+O\left(r^{-1}\right)\right)}{\frac{\pi \exp (-r)}{\sqrt{2 \pi r}}\left(1+O\left(r^{-1}\right)\right)} \rightarrow 1 \\
\frac{I_{\alpha}(r)}{I_{\beta}(r)}=\frac{\frac{\exp r}{\sqrt{2 \pi r}}\left(1+O\left(r^{-1}\right)\right)}{\frac{\exp r}{\sqrt{2 \pi r}}\left(1+O\left(r^{-1}\right)\right)} \rightarrow 1
\end{gathered}
$$

and

$$
r K_{\alpha}(r) I_{\beta}(r)=r \frac{\pi \exp (-r)}{\sqrt{2 \pi r}}\left(1+O\left(r^{-1}\right)\right) \frac{\exp r}{\sqrt{2 \pi r}}\left(1+O\left(r^{-1}\right)\right) \rightarrow \frac{1}{2} .
$$

To prove (2.24), we apply De L'Hospital's rule and calculate instead in both cases the limit of the quotient of derivatives. Using that $I_{0}^{\prime}=I_{1}$ and $K_{0}^{\prime}=-K_{1}$ [9], we thus obtain (2.24) by combining (2.25), (2.26), (2.27) and the fact that

$$
\begin{gathered}
\frac{-K_{0}(r)}{\frac{d}{d r} \frac{1}{r I_{0}(r)}}=\frac{K_{0}(r) I_{0}(r) r}{\frac{I_{1}(r)}{I_{0}(r)}+\frac{1}{r}} \rightarrow \frac{\frac{1}{2}}{1+0}=\frac{1}{2} \\
\frac{I_{0}(r)}{\frac{d}{d r} \frac{1}{r K_{0}(r)}}=\frac{I_{0}(r) K_{0}(r) r}{\frac{K_{1}(r)}{K_{0}(r)}+\frac{1}{r}} \rightarrow \frac{\frac{1}{2}}{1+0}=\frac{1}{2}
\end{gathered}
$$

as $r \rightarrow \infty$. As a byproduct of (2.24) and the equality $K_{0}^{\prime}=-K_{1}$ we obtain that

$$
\frac{d}{d r}\left(r K_{0}(r)\right)=K_{0}(r)\left(1-\frac{K_{1}(r)}{K_{0}(r)} r\right)<0
$$

for large $r$, and so the map $r \mapsto r K_{0}(r)$ is decreasing on $[R, \infty)$ for sufficiently large $R>0$. This implies (2.23) and also that, for $R>0$ large enough, the map $r \mapsto P_{\text {id }}(1, r, R)$ is decreasing on $[R, \infty)$, hence maximal for $r=R$. By $(2.24)$, we see that $P_{\text {id }}(1, R, R) \rightarrow \frac{1}{2}$ as $R \rightarrow \infty$. In view of (2.24) this implies the last inequality in (2.22); the second inequality in (2.22) is trivial, while the first follows from the limit relation (2.21).

In particular, we conclude that all three terms $M_{\mathrm{id}}(1, r), N_{\mathrm{id}}(1, r, R)$, and $I_{0}(R) P_{\text {id }}(1, r, R) / K_{0}(R)$ remain uniformly bounded for large $r$. To see that $M_{\text {id }}(1, r)$ and $I_{0}(R) P_{\text {id }}(1, r, R) / K_{0}(R)$ are uniformly bounded for small $r>R$ as well, it suffices to recall that $K_{0} \in L^{1}([0, \infty))$ and that $I_{0}(R) / K_{0}(R) \rightarrow 0$ as $R \rightarrow 0$. This completes the proof. 
Remark 2.3. Since both limits (2.24) are nonzero, this shows that $w=\mathrm{id}$ is in a sense the only possible choice. In fact, increasing the growth of $w$ seems, by numerical and empirical experiments, to increase the first limit (2.24) (and will usually make it infinite), while decreasing the growth of $w$ will typically increase the second limit (2.24). Hence, in a certain sense we are restricted to linear growth of $w$, i.e., essentially to the choice $w(r)=r$.

Remark 2.4. Numerical experiments suggest that $L \approx 1.2$ is sufficiently small for the estimate (2.20). More precisely, it seems that always $\frac{1}{c^{2}}<L_{\mathrm{id}}(c, R) \leq \frac{L}{c^{2}}$, where the upper bound is attained for $R \approx 0.1$, and $L_{\mathrm{id}}(c, R)$ tends to the lower bound as $R \rightarrow \infty$. In other words, the estimate (2.22) can probably be sharpened to

$$
\lim _{R \rightarrow \infty} L_{\mathrm{id}}(c, R)=\frac{1}{c^{2}}
$$

In fact, in view of (2.19) it suffices to consider the case $c=1$. The following figure which was plotted with the GNU Octave program, shows the behaviour of $M_{\mathrm{id}}(1, R)+N_{\mathrm{id}}(1, r, R)+\frac{I_{0}(R) P_{\mathrm{id}}(1, r, R)}{K_{0}(R)}$ as a function of $r$ for various values of $R$. In accordance with Lemma 2.2, all functions in tend to 1 as $r \rightarrow \infty$. It seems that, for small $R>0$ (less than 0.1 , say), the maxima of these functions are increasing with respect to $R$, while for large $R$ the maxima are decreasing with respect to $R$ and tend to 1 as $R \rightarrow \infty$.

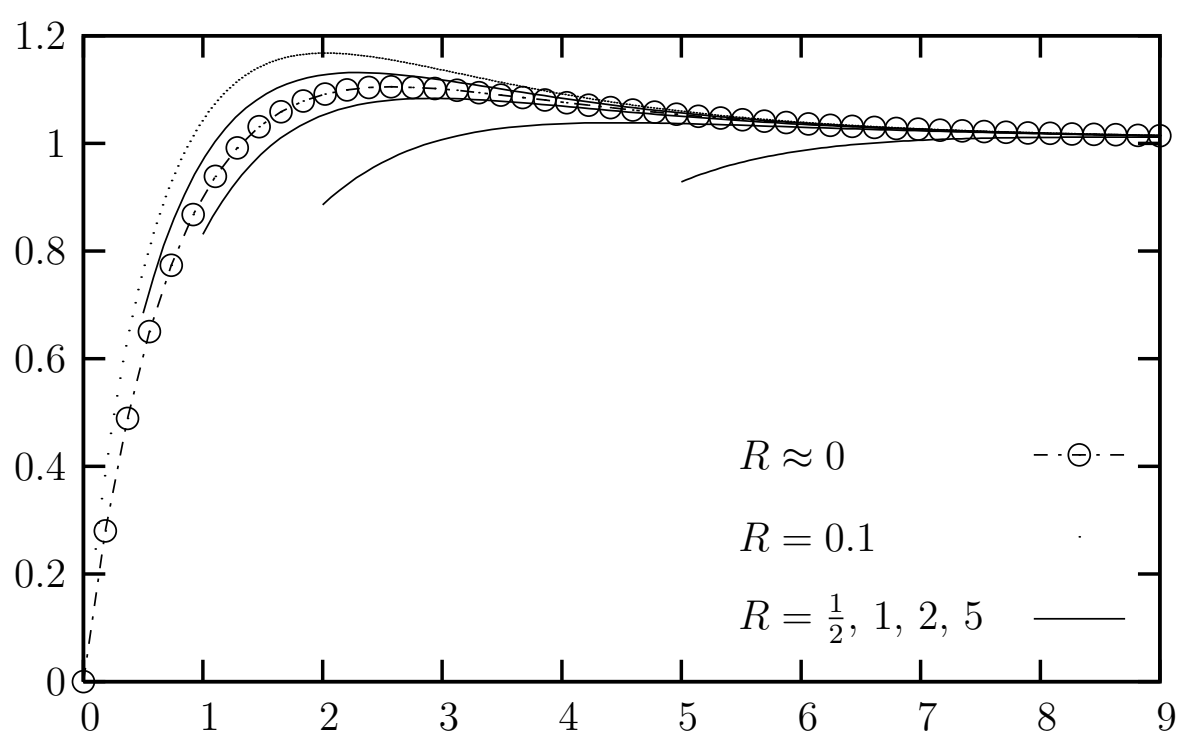

Now we return to the operator $A$ defined by equality (2.14). In particular, we will see why it is crucial to have upper estimates, or even explicit formulas, for the constant (2.15). 
Lemma 2.5. Suppose that $w:[R, \infty) \rightarrow(0, \infty)$ is continuous with $(2.5)$ and (2.23) and has the property that $L_{w}(c, R)<\infty$. Then each $y \in C_{w}([R, \infty))$ satisfies (2.12) and (2.13). Moreover, the operator $A$ maps the space $C_{w}([R, \infty))$ into itself and satisfies a Lipschitz condition with constant $L_{w}(c, R)$ in the norm (2.6).

Proof. Using the shortcut

$$
h(r):=\frac{K_{0}(c r)}{K_{0}(c R)}
$$

for $y \in C_{w}([R, \infty))$ and $r \geq R$ we have

$$
\begin{aligned}
|A y(r) w(r)| \leq & I_{0}(c r) w(r) \int_{r}^{\infty} K_{0}(c t) \frac{t}{w(t)}|y(t) w(t)| d t \\
& +K_{0}(c r) w(r) \int_{R}^{r} I_{0}(c t) \frac{t}{w(t)}|y(t) w(t)| d t \\
& +h(r)\left(\left|u_{0}\right| w(r)+I_{0}(c R) \int_{R}^{\infty} K_{0}(c t) \frac{t}{w(t)}|y(t) w(t)| d t\right) \\
\leq & M_{w}(c, r)\|y\|_{w}+N_{w}(c, r, R)\|y\|_{w}+h(r) w(r)\left|u_{0}\right| \\
& +\frac{I_{0}(c R)}{K_{0}(c R)} P_{w}(c, r, R)\|y\|_{w} \\
\leq & L_{w}(c, R)\|y\|_{w}+h(r) w(r)\left|u_{0}\right| \\
\leq & B_{w}\left(c, R, y, u_{0}\right),
\end{aligned}
$$

where the constant $B_{w}\left(c, R, y, u_{0}\right):=L_{w}(c, R)\|y\|_{w}+\left\|u_{0} h\right\|_{w}$ is finite in view of (2.23). In particular, $A y \in C_{w}([R, \infty))$, and starting from the second estimate, we obtain also

$$
\int_{R}^{\infty} K_{0}(c t)|y(t)| t d t=\int_{R}^{\infty} K_{0}(c t) \frac{t}{w(t)}|y(t) w(t)| d t \leq \frac{B_{w}\left(c, R, y, u_{0}\right)}{I_{0}(c r) w(r)}<\infty
$$

and

$$
K_{0}(c r)\left|\int_{R}^{r} I_{0}(c t) t y(t) d t\right| \leq K_{0}(c r) \int_{R}^{r} I_{0}(c t) \frac{t}{w(t)}|y(t) w(t)| d t \leq \frac{B_{w}\left(c, R, y, u_{0}\right)}{w(r)} .
$$

In particular, (2.12) follows from the first of these inequalities and (2.13), in view of $(2.5)$, from the second. Now, for $x, y \in C_{w}([R, \infty))$, we estimate similarly as above, observing that the inhomogeneous term containing $u_{0}$ drops out for the 
difference, and obtain

$$
\begin{aligned}
& |(A x(r)-A y(r)) w(r)| \\
& \leq I_{0}(c r) w(r) \int_{r}^{\infty} K_{0}(c t) \frac{t}{w(t)}|(x(t)-y(t)) w(t)| d t \\
& \quad+K_{0}(c r) w(r) \int_{R}^{r} I_{0}(c t) \frac{t}{w(t)}|(x(t)-y(t)) w(t)| d t \\
& \quad+\frac{I_{0}(c R)}{K_{0}(c R)} K_{0}(c r) w(r) \int_{R}^{\infty} K_{0}(c t) \frac{t}{w(t)}|(x(t)-y(t)) w(t)| d t \\
& \leq M_{w}(c, r)\|x-y\|_{w}+N_{w}(c, r, R)\|x-y\|_{w}+\frac{I_{0}(c R)}{K_{0}(c R)} P_{w}(c, r, R)\|x-y\|_{w} .
\end{aligned}
$$

Taking the supremum over $r \in[R, \infty)$ we see that $\|A x-A y\|_{w} \leq L_{w}(c, R) \| x-$ $y \|_{w}$ which proves the assertion.

Observe that the hypothesis (2.23) may equivalently be formulated as $h \in$ $C_{w}([R, \infty))$, where $h$ is given by $(2.29)$.

\section{The nonlinear boundary value problem}

Now we take the nonlinearity of $f$ from (2.1) into account. To this end, we will choose in the previous discussion a value $c>0$ which is close to $\sqrt{f^{\prime}(0)}$. In fact, one could always choose $c=\sqrt{f^{\prime}(0)}$, but, depending on the shape of $f^{\prime}$, other choices might be better. The choice of $c>0$ must be made in such a way that

$$
\left|f^{\prime}(0)-c^{2}\right|<\frac{1}{L_{w}(c, R)}
$$

with $L_{w}(c, R)$ given by (2.15). Of course, (3.1) is trivially satisfied for $c=$ $\sqrt{f^{\prime}(0)}$ (if $L_{w}(c, R)<\infty$ ); however, it is desirable for our results (see Theorems 3.2 and 3.3 below) that the derivative of the function $f_{c}$ defined by

$$
f_{c}(u):=f(u)-c^{2} u
$$

is not necessarily zero at $u=0$, but has the following special property. For $0<$ $m<L_{w}(c, R)$ let us denote by $I_{f}(c, m)$ the maximal (and thus automatically closed) interval which contains 0 and satisfies

$$
\sup \left\{\left|f_{c}^{\prime}(u)\right|: u \in I_{f}(c, m)\right\} \leq m .
$$

We then have to choose $c$ in such a way that $I_{f}(c, m)$ is, in a certain sense, large for $m$ close to $\frac{1}{L_{w}(c, R)}$, and to this end more sophisticated choices than just $c=\sqrt{f^{\prime}(0)}$ might be preferable. Observe that, for any choice of $c>0$ with (3.1), 0 is an interior point of the interval $I_{f}(c, m)$ if $m$ is close enough to $\frac{1}{L_{w}(c, R)}$. 
For $w, f, c$ and $m$ as above, consider the set

$$
C_{w, f}(c, m):=\left\{u \in C_{w}([R, \infty)): u([R, \infty)) \subseteq I_{f}(c, m)\right\},
$$

endowed with the metric inherited from the norm (2.6). Obviously, the interval $I_{f}(c, m)$ increases with increasing $m$, and so the same is true for the set $C_{w, f}(c, m)$. For each $m>0$, the function $f_{c}$ induces a nonlinear Nemytskij operator $F_{c}: C_{w, f}(c, m) \rightarrow C_{w}([R, \infty))$ by the formula

$$
F_{c}(u)(r):=f_{c}(u(r)) .
$$

The next lemma shows that this operator as well satisfies a Lipschitz condition with respect to the norm (2.6).

Lemma 3.1. The operator $F_{c}$ maps $C_{w, f}(c, m)$ into $C_{w}([R, \infty))$ and satisfies a Lipschitz condition with Lipschitz constant $m$.

Proof. For all $u, v \in C_{w, f}(c, m)$ and all $r \in[R, \infty)$, we have $u(r), v(r) \in I_{f}(c, m)$, and so the mean value theorem implies $\left|f_{c}(u(r))-f_{c}(v(r))\right| \leq m|u(r)-v(r)|$. Hence,

$$
\left|\left(F_{c}(u)-F_{c}(v)\right)(r) w(r)\right| \leq m|(u(r)-v(r)) w(r)| \leq m\|u-v\|_{w},
$$

and so $\left\|F_{c}(u)-F_{c}(v)\right\|_{w} \leq m\|u-v\|_{w}$. Since $f(0)=0$ implies $f_{c}(0)=0$, and thus $F_{c}(0)=0$, the above calculation with $v=0$ shows also that $\left\|F_{c}(u)\right\|_{w}<\infty$, i.e., $F_{c}(u) \in C_{w}([R, \infty))$.

Now we are in a position to prove our main uniqueness result for the nonlinear boundary value problem $(2.1) /(2.2)$. To this end, we consider the set

$$
C_{w, f, c, R}:=\bigcup_{0<m<\frac{1}{L w(c, R)}} C_{w, f}(c, m) .
$$

Theorem 3.2. Suppose that the hypotheses of Lemma 2.5 are satisfied, and that (3.1) holds. Then the nonlinear boundary value problem $(2.1) /(2.2)$ has at most one solution in the set (3.4). In particular, if $f^{\prime}(0)>0$, then there is at most one solution in the set

$$
\bigcup_{0<m<\frac{f^{\prime}(0)}{L}} C_{\mathrm{id}, f}\left(\sqrt{f^{\prime}(0)}, m\right),
$$

where $L$ is the universal constant from Lemma 2.2 .

Proof. By Lemma 2.2, the set $C_{\mathrm{id}, f, \sqrt{f^{\prime}(0)}, R}$ contains the set (3.5). To prove uniqueness in the set (3.4), let $u, v \in C_{w, f, c, R}$ be two solutions of $(2.1) /(2.2)$. Since the sets $C_{w, f}(c, m)$ increase with increasing $m$, we may assume without loss of generality that $u, v \in C_{w, f}(c, m)$ for the same constant $m \in\left(0, \frac{1}{L_{w}(c, R)}\right)$. 
Moreover, since both $F_{c}(u)$ and $F_{c}(v)$ belong to $C_{w}([R, \infty))$, by Lemma 3.1, from Proposition 2.1 and Lemma 2.5 we conclude that $u=A F_{c}(u)$ and $v=$ $A F_{c}(v)$, with $A$ given by (2.14). Consequently, Lemma 2.5, Lemma 3.1, and Proposition 2.1 imply

$\|u-v\|_{w}=\left\|A F_{c}(u)-A F_{c}(v)\right\|_{w} \leq L_{w}(c, R)\left\|F_{c}(u)-F_{c}(v)\right\|_{w} \leq L_{w}(c, R) m\|u-v\|_{w}$.

Since $0<L_{w}(c, R) m<1$, this implies that $\|u-v\|_{w}=0$, i.e., $u=v$ as claimed.

We point out that the hypotheses of Theorem 3.2 and the following Theorem 3.3 are satisfied, e.g., for $w=$ id and $c=\sqrt{f^{\prime}(0)}\left(\right.$ if $f^{\prime}(0)>0$ ). In the following section we will give two examples how to calculate the sets (3.4) and (3.5) in this case.

We formulate now a corresponding existence and approximation result which is based on Banach's contraction mapping theorem. The proof of Theorem 3.2 suggests to choose $q:=m L_{w}(c, R)$ as a contraction constant. For $r \geq R$, let

$$
\rho:=\frac{q}{1-q} \sup _{r \geq R} \frac{K_{0}(c r)}{K_{0}(c R)} w(r)=\frac{q}{1-q}\|h\|_{w}
$$

where $h$ is defined as in (2.29). In the next theorem, we choose the initial value $u_{0}$ from $(2.2)$ in such a way that

$$
\left|u_{0}\right| \rho \leq \inf _{r \geq R} w(r) \operatorname{dist}\left(u_{0} h(r), \mathbb{R} \backslash I_{f}(c, m)\right),
$$

where $I_{f}(c, m) \subseteq \mathbb{R}$ is the interval occurring in definition (3.3). Condition (3.7) may certainly be achieved if $u_{0}$ is sufficiently small. Geometrically, (3.7) means that, roughly speaking, a good choice for $c$ should give an interval $I_{f}(c, m)$ with the property that, for $m$ close to $\frac{1}{L_{w}(c, R)}$, the values of the function $\left|u_{0}\right| h$ remain "in the middle" of this interval. If this interval is symmetric, i.e., $I_{f}(c, m)=[-\Lambda, \Lambda]$, then the "middle" yields of course a condition on the difference $\Lambda-\left|u_{0}\right| h(r)$. Since $h$ is monotonically decreasing, the distance function in (3.7) is monotonically increasing in $r$. If, in addition, the weight function $w$ is also increasing, the infimum in (3.7) is attained for $r=R$; so in this case condition (3.7) simply reads

$$
\left|u_{0}\right| \frac{q}{1-q}\|h\|_{w} \leq w(R)\left(\Lambda-\left|u_{0}\right|\right) .
$$

In other words, in this case the initial value $\left|u_{0}\right|$ should be left of the middle of the interval $[0, w(R) \Lambda]$, where the "middle" is weighted by the other data. We will come back to this, for the particular case $w(r)=r$, in Section 4 . 
Theorem 3.3. Suppose that the hypotheses of Lemma 2.5 are satisfied and that $\left|f^{\prime}(0)-c^{2}\right|<m<\frac{1}{L_{w}(c, R)}$. Assume that either $I_{f}(c, m)=\mathbb{R}$ or $(3.7)$ is true. Then the nonlinear boundary value problem $(2.1) /(2.2)$ has a unique solution $u^{*} \in C_{w, f}(c, m)$. More precisely, the closed ball

$$
B:=\left\{u \in C_{w}([0, \infty)):\left\|u-u_{0} h\right\|_{w} \leq\left|u_{0}\right| \rho\right\},
$$

with $h(r)$ given by (2.29) and $\rho$ given by (3.6), is contained in the class $C_{w, f}(c, m)$. Moreover, the operator $A F_{c}$ maps this ball into itself and satisfies a Lipschitz condition with Lipschitz constant $q=m L_{w}(c, R) \in(0,1)$. The solution $u^{*}$ is the unique fixed point of $A F_{c}$ in $B$ and, for each $u \in B$, the successive approximations $\left(A F_{c}\right)^{n}(u)$ converge to the solution $u^{*} \in B$ and satisfy the a priori estimate

$$
\left\|u^{*}-\left(A F_{c}\right)^{n}(u)\right\|_{w} \leq \frac{q^{n}}{1-q}\left\|A F_{c}(u)-u\right\|_{w} .
$$

Proof. We show first the inclusion $B \subseteq C_{w, f}(c, m)$. In case $I_{f}(c, m)=\mathbb{R}$ we have $C_{w, f}(c, m)=C_{w}([R, \infty))$, and so there is nothing to prove. Thus, assume that $I_{f}(c, m)$ does not coincide with the real line and that (3.7) holds. For all $u \in B$ and all $r \in[R, \infty)$, we have

$$
\left|u(r)-u_{0} h(r)\right|=\frac{\left|\left(u(r)-u_{0} h(r)\right) w(r)\right|}{w(r)} \leq \frac{\left\|u-u_{0} h\right\|_{w}}{w(r)} \leq \frac{\left|u_{0}\right| \rho}{w(r)} .
$$

Since $\left|u_{0}\right| \rho$ can be estimated by the right hand side of (3.7), we have $\left|u_{0}\right| \rho \leq$ $w(r) \operatorname{dist}\left(u_{0} h(r), \mathbb{R} \backslash I_{f}(c, m)\right)$, and combining this with (3.11) we obtain

$$
\left|u(r)-u_{0} h(r)\right| \leq \operatorname{dist}\left(u_{0} h(r), \mathbb{R} \backslash I_{f}(c, m)\right) .
$$

Since $I_{m}(c, f)$ is closed, this shows that $u(r) \in I_{f}(c, m)$, and so $u \in C_{w, f}(c, m)$, as required.

So from Lemma 3.1 we conclude that $F_{c}: B \rightarrow C_{w}([R, \infty))$ satisfies a Lipschitz condition with Lipschitz constant $m$. In view of Lemma 2.5, the operator $A F_{c}: B \rightarrow C_{w}([R, \infty))$ thus satisfies a Lipschitz condition with Lipschitz constant $q$. For all $u \in B$, we have $\|u\|_{w} \leq\left\|u_{0} h\right\|_{w}+\left|u_{0}\right| \rho$, which in view of $u_{0} h=A(0)=A F_{c}(0)$ implies the estimate

$$
\left\|A F_{c}(u)-u_{0} h\right\|_{w}=\left\|A F_{c}(u)-A F_{c}(0)\right\|_{w} \leq q\|u-0\|_{w} \leq q\left(\left|u_{0}\right| \rho+\left\|u_{0} h\right\|_{w}\right) .
$$

Now observe that $q\left\|u_{0} h\right\|_{w}=(1-q)\left|u_{0}\right| \rho$, by (3.6); combining this with (3.12) we conclude that, for each $u \in B$, the estimate

$$
\left\|A F_{c}(u)-u_{0} h\right\|_{w} \leq q\left|u_{0}\right| \rho+q\left\|u_{0} h\right\|_{w}=q\left|u_{0}\right| \rho+(1-q)\left|u_{0}\right| \rho=\left|u_{0}\right| \rho
$$

holds true, i.e., $A F_{c}$ maps indeed the ball $B$ into itself. By Banach's fixed point theorem, the operator $A F_{c}$ has a unique fixed point $u^{*} \in B$ which may be approximated as described in the claim. Since $y=F_{c}\left(u^{*}\right)$ belongs to $C_{w}([R, \infty))$, 
Proposition 2.1 implies, in view of Lemma 2.5, that $u^{*}=A F_{c}\left(u^{*}\right)$ is a solution of $(2.1) /(2.2)$. By Theorem 3.2, the solution is even unique in the larger set $C_{w, f}(c, m)$.

Remark 3.4. As mentioned at the beginning of this section, one can usually expect much faster convergence in (3.10) if $u^{*}$ is very small. In fact, if $u_{n}:=$ $\left(A F_{c}\right)^{n}(u)$ gets smaller, one may apply the result again with $u_{n}$ in place of $u$, but then one may choose $m$ and thus $q$ also smaller. In this way, it is possible to improve the original a priori estimate in each step. The reason for this is that our algorithm is in fact a simplified Newton method corresponding to the linearization of the problem at 0 , and so one may typically expect almost quadratic convergence if the solution $u^{*}$ is close to the point of linearization.

\section{The example from Section 1 revisited}

Now we analyze our results for the case of the functions (2.3) and (2.4) which arise naturally in the application discussed in Section 1 and both satisfy $f(0)=0$. Since $f^{\prime}(0)=\alpha a>0$ for the function (2.3), and similarly $f^{\prime}(0)=\alpha(a+b)>0$ for the function (2.4), we may apply the results of the preceding sections.

We consider $w:=\mathrm{id}$ and are looking for "optimal choices" for $c>0$ and $m \in\left(0, \frac{1}{L_{\mathrm{id}}(c, R)}\right)$. For simplicity, we impose on $m$ the slightly more restrictive assumption

$$
\lambda:=\frac{c^{2}}{m} \in(L, \infty)
$$

where $L>1$ is the universal constant from Lemma 2.2.

We start with the function (2.3). The shifted function (3.2) is here $f_{c}(u)=$ $\alpha \sinh (a u)-c^{2} u$, and so $I_{f}(c, m)$, see $(3.3)$, is the largest interval around 0 containing all solutions $u$ of the inequality

$$
c^{2}-m \leq a \alpha \cosh (a u) \leq c^{2}+m .
$$

Since the middle term in (4.2) depends only on $|u|$ and is increasing with respect to $|u|$, the left inequality is satisfied if and only if it is satisfied for $u=0$. If, in addition, the right inequality is satisfied for $u=0$, then the solution set of (4.2) is automatically a symmetric interval of the form $[-\Lambda(\lambda), \Lambda(\lambda)]$. Therefore we have to require $c^{2}-m \leq a \alpha \leq c^{2}+m$, i.e.,

$$
(\lambda-1) m \leq f^{\prime}(0) \leq(\lambda+1) m .
$$

Conversely, if (4.3) holds then, since the function cosh: $[0, \infty) \rightarrow[1, \infty)$ is a bijection with inverse $\cosh ^{-1} t=\log \left(t+\sqrt{t^{2}-1}\right)$, we obtain that $I_{f}(c, m)=$ $[-\Lambda(\lambda), \Lambda(\lambda)]$ is symmetric with

$$
\begin{aligned}
\Lambda(\lambda) & :=\frac{1}{a} \log \frac{1}{a \alpha}\left(c^{2}+m+\sqrt{\left(c^{2}+m\right)^{2}-a^{2} \alpha^{2}}\right) \\
& =\frac{1}{a} \log \frac{1}{f^{\prime}(0)}\left((\lambda+1) m+\sqrt{(\lambda+1)^{2} m^{2}-f^{\prime}(0)^{2}}\right) .
\end{aligned}
$$


The larger we can choose $\Lambda(\lambda)$, the stronger becomes the uniqueness statement of Theorem 3.2. For fixed $\lambda>L$, we obtain the maximal value of $\Lambda(\lambda)$ if we choose $m$ maximal, i.e., such that we have equality in the left estimate of (4.3). Solving this equation for $m$ (the right estimate of (4.3) holds in this case automatically), and inserting this choice of $m$ into (4.4) we obtain (for fixed $\lambda>L$ ) the optimal value

$$
\Lambda(\lambda)=\frac{1}{a} \log \left(\frac{\lambda+1+2 \sqrt{\lambda}}{\lambda-1}\right)=\frac{1}{a} \log \frac{\sqrt{\lambda}+1}{\sqrt{\lambda}-1} .
$$

Since $x \mapsto \frac{x+1}{x-1}$ is strictly decreasing, the function $\lambda \mapsto \Lambda(\lambda)$ is also strictly decreasing. For an appropriate choice of $\lambda \in(L, \infty)$ we thus may get this expression as close to the limit case $\lambda=L$ as we want. Consequently, Theorem 3.2 and Lemma 2.2 imply the following uniqueness result.

Theorem 4.1. Let $f$ be the function (2.3) with a, $\alpha>0$, and let $L \approx 1.2$ be as in Lemma 2.2. Then the boundary value problem $(2.1) /(2.2)$ has at most one solution $u$ satisfying $u(r)=O\left(\frac{1}{r}\right)$ as $r \rightarrow \infty$ and

$$
|u(r)|<\frac{1}{a} \log \frac{\sqrt{L}+1}{\sqrt{L}-1} \approx \frac{3}{a} \quad(R \leq r<\infty) .
$$

Our calculations indicate that, if we require (3.1) and want to apply Theorem 3.2, we cannot improve the constant in Theorem 4.1. Concerning Theorem 3.3, we do not get necessarily the best result if we maximize $\Lambda(\lambda)$, because also (3.8) must be satisfied, i.e.,

$$
\left|u_{0}\right| \leq\left(\frac{q}{1-q}\|h\|+R\right)^{-1} R \Lambda(\lambda)
$$

where $q$ also depends on our parameters $m$ and $c$, hence on $\lambda$. We apply again Lemma 2.2 and use the estimate $q \leq \frac{m L}{c^{2}}=\frac{L}{\lambda}$ with $L$ as before. Since the right hand side of (4.5) decreases with $q$, we obtain a sufficient condition for (4.5) when we replace $q$ by $\frac{L}{\lambda}$. Hence, concerning Theorem 3.3, we should aim at choosing the parameters $c$ and $m$ with (4.1) and (4.3) such that the right hand side of the (sufficient) condition

$$
\left|u_{0}\right| \leq\left(\frac{L}{\lambda-L}\|h\|+R\right)^{-1} R \Lambda(\lambda)
$$

becomes maximal. Assuming for a moment for simplicity that $\|h\|$ is a constant (although it actually might vary slightly with $c$ ) then, for fixed $m$, this value becomes maximal if $\lambda$ is maximal. This heuristic argument suggests that, for fixed $m$, we should strive to to choose $\lambda$ as large as possible, i.e. such that we have equality in the left estimate of (4.3). Solving this equality for $m$ (for this 
choice the right estimate of (4.3) holds automatically), and inserting into (4.6), we obtain for the right hand side of (4.6) the expression

$$
B_{R}(\lambda):=\frac{R}{a}\left(\frac{L}{\lambda-L}\|h\|+R\right)^{-1} \log \frac{\sqrt{\lambda}+1}{\sqrt{\lambda}-1} .
$$

Our choices for $m$ and $c$ allow us to take the largest initial value $u_{0}$ in (4.6) if $\lambda \in$ $(L, \infty)$ maximizes $B_{R}(\lambda)$. Note that $\|h\|$ depends continuously on $c$ (and such on $\lambda$ ) and that $B_{R}(\lambda) \rightarrow 0$ as $\lambda \rightarrow L$ or $\lambda \rightarrow \infty$ so that this maximum indeed exists. Recalling that we have chosen $m$ according to $(\lambda-1) m=f^{\prime}(0)=a \alpha$ and $c$ according to (4.1), we thus obtain with Theorem 3.3 the following existence result.

Theorem 4.2. Let $f$ be the function (2.3) with $a, \alpha>0$, and let $L \approx 1.2$ be as in Lemma 2.2. For $R>0$, let

$$
B(R):=\max _{L<\lambda<\infty} B_{R}(\lambda)
$$

where $B_{R}(\lambda)$ is given by (4.7). Then for each $u_{0} \in[-B(R), B(R)]$ the boundary value problem $(2.1) /(2.2)$ has a solution in $C([R, \infty))$ which can be obtained by the method of successive approximations, provided that the iteration starts from the solution $u_{0} h$, with $h$ given by (2.29), of the linearized problem. More precisely, for each $\lambda \in(L, \infty)$ and each $u_{0} \in\left[-B_{R}(\lambda), B_{R}(\lambda)\right]$, the conclusion of Theorem 3.3 holds with $w:=$ id and with the numbers

$$
m:=\frac{a \alpha}{\lambda-1}, \quad c:=\sqrt{\frac{\lambda a \alpha}{\lambda-1}}, \quad q:=\frac{L}{\lambda}, \quad \rho:=\frac{L}{\lambda-L}\|h\| .
$$

Again, our calculations indicate that, if we want to apply Theorem 3.3 and do not use finer estimates for $L_{\text {id }}(c, R)$ than (2.20), the constants in Theorem 4.2 are at least close to the best possible.

To conclude we make some remarks on the function (2.4). In a physical interpretation, the nonsymmetric function (2.4) which contains one more degree of freedom than (2.3) corresponds to a multi-component electrolyte and has therefore some physical interest. However, the mathematical treatment becomes much more involved than in case of the sinh function (2.3). In fact, for (2.3) we obtained the explicit form of the interval $I_{f}(c, m)$ simply by solving a quadratic equation, see (4.4). Substituting $\beta:=\frac{b}{a}$ in (2.4) gives $f(u)=\alpha\left(e^{a u}-e^{-\beta a u}\right)$, and so $I_{f}(c, m)$ is the largest interval around 0 containing all solutions $u$ of the inequality

$$
c^{2}-m \leq a \alpha\left(e^{a u}+\beta e^{-\beta a u}\right) \leq c^{2}+m .
$$

Putting $e^{a u}=: x$ we obtain from (4.8) the algebraic inequality

$$
c^{2}-m \leq a \alpha\left(x+\beta x^{-\beta}\right) \leq c^{2}+m .
$$


Of course, in case $b=a$, i.e., $\beta=1$, multiplying by $x$ we get the previous quadratic equation for $x$, which led to the above calculations for the function (2.3). However, even if $\beta>1$ is an integer, multiplying (4.9) by $x^{\beta}$ we end up with a polynomial equation of degree $\beta+1>2$ for which usually a closed solution is not known. In other words, in case of the function (2.4) with specific parameters $a, b$ and $\alpha$, one can expect only rough numerical upper and lower bounds for the interval $I_{f}(c, m)$, if there are any, but not closed formulas as in the symmetric case $(2.3)$.

Acknowledgement. The paper was written in the framework of a joint DAADNSC project (Kz. 423/rc-PPP-Sr), while the first and last authors were visiting Tamkang and Tunghai University. The last author is a Heisenberg fellow (Az. VA 206/1-2). Financial support by the DAAD (Deutscher Akademischer Austausch-Dienst), NSC (National Science Council of Taiwan) and DFG (Deutsche Forschungsgemeinschaft) is gratefully acknowledged.

\section{References}

[1] Hsu, J.-P., Jiang, J.-M. and Tseng, S., Electrical interaction between two rodlike particles covered by an ion-penetrable membrane in a water-oil interface. Coll. Surf. B-27 (2003), $49-58$.

[2] Hunter, R. J., Foundations of Colloid Sciences. London: Oxford Univ. Press 1989.

[3] Korenev, B. G., Introduction to the Theory of Bessel Functions (in Russian). Moscow: Nauka 1971; Engl. transl.: London: Taylor \& Francis 2002.

[4] Luke, Y. L., Integrals of Bessel Functions. New York: McGraw-Hill 1962.

[5] McGhee, D. F., Madbouly, N. M. and Roach, G. F., Applications of fixed point theorems to a chemical reactor problem. In: Integral Methods in Science and Engineering (Proc. 7th Intern. Conf. IMSE, Saint Étienne 2002; ed.: C. Constanda). Basel: Birkhäuser 2004, pp. 133 - 138.

[6] Olver, F. W. J., Introduction to Asymptotics and Special Functions. New York: Academic Press 1974.

[7] Petiau, G., La théorie des fonctions de Bessel exposée en vue de ses applications à la physique mathématique. Paris: Centre Nat. Rech. Sci. 1955.

[8] Tseng, S., Jiang, J.-M. and Hsu, J.-P., Electrical potential in a cylindrical double layer: a functional theory approach. J. Coll. Interface Sci. 273 (2004), $218-223$.

[9] Watson, G. N., A Treatise on the Theory of Bessel Functions. Cambridge: Cambridge Univ. Press 1944.

Received May 24, 2006 